\title{
PREVENTION OF WATER INGRESS IN HYDRAULIC SYSTEMS
}

\author{
Samo Goljat ${ }^{1 *}$, Vito Tič ${ }^{1}$, Darko Lovrec ${ }^{1}$ \\ ${ }^{1}$ University of Maribor, Faculty of Mechanical Engineering, Maribor, Slovenia
}

\begin{abstract}
:
Industrial and mobile hydraulic systems are exposed to various environmental impacts. In order to achieve reliable operation and stable long-term use, we have to prevent ingress of different contaminants into hydraulic fluid and keep contamination levels on minimum. One of most common contaminants is water, which can significantly affect life-time of a hydraulic system. Thus, special attention should be paid to keep water content and humidity of hydraulic oil on lowest possible level, especially when using particular types of oil.

The paper investigates different approaches on how to efficiently monitor water contamination and prevent water ingress into hydraulic system.
\end{abstract}

\section{ARTICLE HISTORY}

Received: 17.06.2021.

Accepted: 01.09.2021.

Available: 30.09.2021.

\section{KEYWORDS}

Hydraulic system, water, contamination, humidity, maintenance, monitoring

\section{INTRODUCTION}

Water contamination in hydraulic oil has been known to cause range of problems in hydraulic systems, resulting in failures and breakdowns. The solution to water contamination can be either water prevention or active water removal systems. In this paper we will focus on water prevention methods. Some of these methods include seal management, reservoir breathers, cooler maintenance mentioned in research work [1], others are i.e. volume compensator made from inflatable bladder which prevents air into coming with contact with hydraulic liquid [2]. In our paper we also mentioned Silica gel filter, which is used in our own centralized hydraulic system for drying air and removing other particles down to $2 \mu \mathrm{m}$ size.

Water can be present in different forms, as seen in Fig.1:

- Dissolved water - Water dissolved in hydraulic fluid at molecular level. It occurs before saturation point of hydraulic fluid is reached. Certain additives and certain kinds of hydraulic fluids can increase the volume of water the oil can dissolve before it reaches point of saturation.

- Emulsified Water - Emerges beyond hydraulic fluid saturation point.
- Free form water - separated phase, can also appear in puddles. This section emphasizes the importance of the research and hitherto research in the given filed.
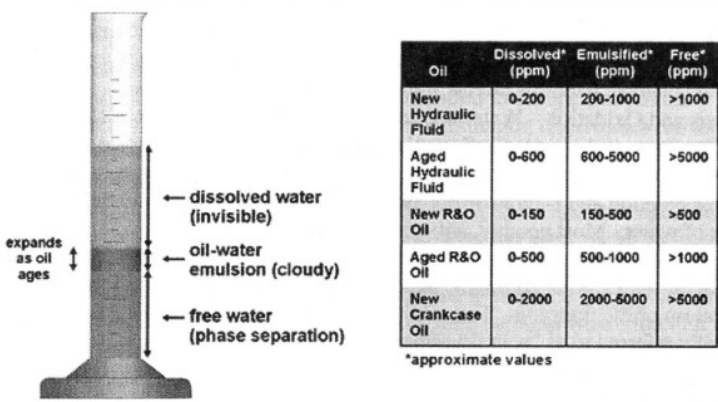

Fig. 1. Water contamination forms present in hydraulic fluid [1]

Saturation occurs when more dissolved water is present than hydraulic fluid can hold. Excessive water can then appear as free water or as an emulsion. For example, free form water is commonly linked to surface corrosion [3]. In research work [3] it was investigated the effects of dissolved water in the fluid can have on a bearing useful life-time. They have discovered that bearing life-time expectancy can be extended many times dependant on how much water was present in lubricant. Test was designed with minimum 
amount of water present in the SAE20 lubricating oil (20 ppm) compared to almost maximum amount of water present before saturation of the SAE20 lubricating oil.

Presence of water in hydraulic fluid causes reduced lubricating characteristics, which can result in increased component wear. There is no standard recommendation, but source suggest [4] that maximum percentage of water present in oil should not exceed 0.05 to $3.0 \%$ of hydraulic system filled volume. When the oil contamination exceeds recommended values, hydraulic components can suffer from slow response, short service life and poor accuracy. Russian statistics show that $20 \%$ break-downs in aviation industry are caused by hydraulic oil contamination [5]. It is recommended that hydraulic system has no water contamination or it is kept to minimum. Even small percentage of water contamination can alter chemical properties of hydraulic fluid and effect surfaces of hydraulic system components, since presence of water affects and degrades hydraulic fluid.

Source of water contamination can be either external or internal. External sources of water contamination are for example: worn seals, condensation from air, seepage through reservoir, rain leakages, topping up impure liquid, inefficient or damaged vent filters. Some of internal sources of water contamination can be leaky heat exchangers or coolers, improper rinsing of the device, etc. [4]. From design point it is also important to separate from absolute humidity and relative humidity. Absolute humidity stays same under different operating conditions and it is expressed in ppm, which means parts per million. On the other side relative humidity tells us how much water is in the air, compared to how much it could hold at that temperature. This means designer of hydraulic system must be careful to predict operating system temperatures in order to prevent hydraulic fluid from reaching $100 \%$ saturation, which causes water to emulsify. For example, if certain hydraulic fluid absolute humidity is $300 \mathrm{ppm}$, that relative humidity at 30 ${ }^{\circ} \mathrm{C}$ can be for example $100 \%$, but at $50{ }^{\circ} \mathrm{C}$ it can only be $60 \%$, which means, that with rise of the temperature fluid can hold more dissolved water. Different hydraulic fluid can also hold different amount of absolute humidity. It is important, that hydraulic system is designed in the way water contamination doesn't exceed maximum relative target moisture levels. For target moisture levels, it is necessary to consider factors e.g. operating temperature of system, type of fluid used, desired absolute and relative moisture levels.

Some fluids, e.g. fire-resistant phosphate-ester fluids used in commercial airplanes, tend to be hygroscopic in nature. Hygroscopic fluids are prone to accumulating humidity from the environment, most commonly from damaged seals and airpressurized reservoirs. When hygroscopic fluid is used, system must be well designed and monitored to prevent water contamination. Water contamination in hygroscopic hydraulic fluids can lead to break down of base fluids into smaller molecular fragments and unwanted by-products. Fragments support autocatalytic reactions, speeding up degradation process and also tending to corrode all kinds of metallic materials inside of a hydraulic system [6]. High moisture levels can also cause foaming, which decreases the efficiency of the system. Foam can create air pockets which are then sucked in by the pump instead of pure fluid. This causes gaps in the fluid film layer, leading to increased wear, because of metal to metal contact.

Research [1] reveals, that by halving water contamination levels in hydraulic fluid, we can reduce hydraulic pump wear rate by $20-50 \%$ and also reduce wear of other hydraulic components. Therefore different water contamination monitoring and preventing methods are used [5].

\section{MEASURING WATER CONTAMINATION IN HYDRAULIC SYSTEM}

Water contamination is most commonly measured by absolute water content expressed in ppm, which stands for parts per million, either by volume or weight. General rule of thumb is, that benefits of monitoring and ensuring maximum target level of moisture in hydraulic system should outweigh the costs of the investment. Research [1] mentions it can be up to about 10 times more expensive to remove water, than to exclude it. Each hydraulic system can differentiate in terms of design, operating conditions, fluid type, therefore optimal maximum target level of moisture can differ from one to another hydraulic system. Most optimal way to determine maximum target level of moisture is hydraulic component supplier's manual, if such a recommendation is available.

There are several water and moisture monitoring methods available. Karl Fischer Titration (ASTM D-1744) is effective way to measure water present in ppm (parts per million) or percent. It should be noted, that certain antiwear, rust inhibiting and EP additives may alter 
test result, resulting in error. It works by inserting Karl Fischer reagent into sample, where electrode is placed. lodine, present in Karl Fischer reagent, reacts with water. When no water is present, the electrodes are depolarized by the iodine, resulting in induced current flow, signalling end of the test [1].

Distillation Method (ASTM D-95) consist of mixing equal amount of solvent and sample fluid. Solvent must be miscible with the sample fluid, but not with water. Mixture of both liquids is poured into the same flask. The solvent and water are then boiled out of the flask, condensed and collected into collection tube. Water concentration is calculated from collected condense [1].

In infrared monitoring method molecules are absorbing energy from beam of infrared light. Energy in molecules is detected at specific frequencies, from which we can determine water contamination. Infrared method has few limitations, such as maximum measurement up to 1000 ppm of water, inaccurate repeatability and possible error caused by additives and contaminants [1].

Less reliable method is crackle test, which consists of dropping droplets on heated surface with temperature ranging from cca. $150{ }^{\circ} \mathrm{C}$ to $160{ }^{\circ} \mathrm{C}$. When emulsified or free water is present, we can see visible and hear audible "crackling" sounds.

Water and moisture detecting techniques mentioned above are offline. In certain areas, e.g. in aviation industry [6], airliners have requested for online monitoring system. Offline monitoring system leads to longer maintenance time in case water is detected and needs to be either extracted from hydraulic fluid or hydraulic fluid needs to be exchanged. Longer maintenance time consequently affects costs related to flight delay. Research [6] mentioned opto-chemical sensor technology, which can be alternative to commonly used method for measuring water contamination/humidity. Commonly used sensor for measuring water contamination/humidity works on electrical principle, detecting electrical properties of hydraulic liquid. Mentioned methods allow continuously online monitoring of water contamination, without collecting samples and sending them to distant laboratories for off-site chemical analysis. Online monitoring approach allows faster fluid degradation diagnostics, resulting in better optimization of necessary maintenance, reducing or preventing need to interrupt normal flight schedules.

\section{WATER EXCLUDING METHODS}

Water removal methods begin with establishing appropriate moisture levels in each hydraulic system. Research [1] has mentioned in his paper, if bearing normal life-time is 1000 hours at 500 ppm water, reducing water to $250 \mathrm{ppm}$ would increase bearing life to about 1500 hours. By further reducing moisture level to about $125 \mathrm{ppm}$, it would increase bearing life-time further to about 2300 hours of service-life.

There are different methods and guidelines to exclude water contamination from hydraulic fluid. One of such is that new hydraulic fluid is kept in environment, which minimizes possibility of water contamination. New hydraulic fluid should be filtered by a moisture-stripping device, before topping-up hydraulic system. It is necessary step, because even new hydraulic fluid can be water contaminated above target moisture levels.

Moisture can get inside the tank through exchange of air with the environment. Hydraulic system should be therefore efficiently sealed and regularly inspected for cracks, damaged seals, etc.

Hydraulic system exchange of air with environment can be solved either by using expansion chambers, desiccant breathers or air conditioners. Normal air filter/breather are not sufficient enough from keeping the air dry, therefore special desiccant breather in our case Silica type gel filter (air conditioner) must be used, which structure is seen on Fig.2 [4].
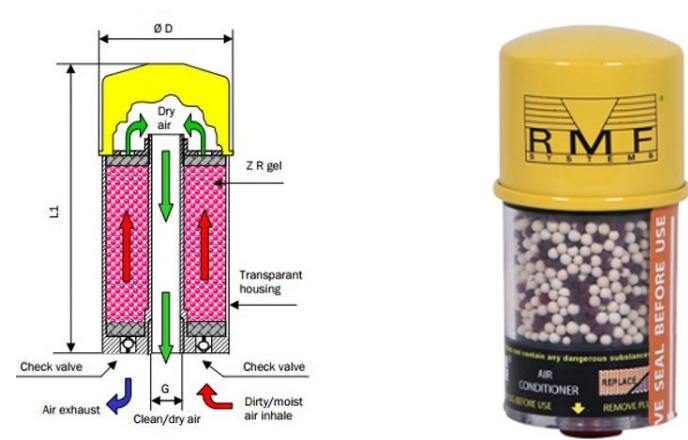

Fig. 2. Structure on left and close up look on right of typical Silica Gel filter

Silica gel inside the filter is used as drying material. Sometimes Silica gel is also combined with active carbon, which removes unpleasant odors. When Silica gel becomes saturated with moisture, the gel itself or indicators in the gel change colour. Fig. 3 shows practical example of silica gel usage on an industrial hydraulic tank. Desiccant breathers are available from several manufacturers and in many sizes. Features include 
small to very large flow rates, miniature sizes, odor and fume removal [7]. These breathers also contain a filter to trap not only moisture, but also particles present in air, down to $2 \mu \mathrm{m}$ filtration, according to source.

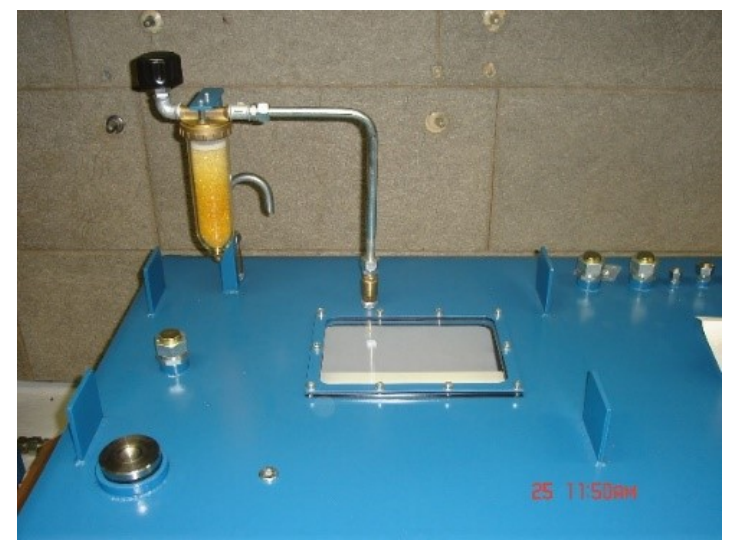

Fig. 3. Operation and appearance of an air dryer (upper), real world example installation on an industrial hydraulic tank (bottom)

The use of a silica gel filter is a classic form of "isolation" of the inside of the tank from the contaminated environment. Usually, the silica gel filter is combined with a non-return valve mounted in parallel, which allows the tank to "inhale" through the silicate gel filter and "exhale" through the non-return filter. This solution is also the cheapest and does not complicate the tank design.

Previously mentioned expansion chambers are also one of the solutions, which can completely prevent ingress of moisture from environmental air into reservoir. Airbag inserted into the tank seen on Fig.4 is one example of expansion chamber. Airbag pre-filled with gas pressure serve as compensating component of hydraulic system for the fluid level fluctuations. The airbag is commonly filled with a pressure of 0.007 to 0.07 bar ( 0.1 to $1 \mathrm{psi})$. Such solution is rather more expensive then use of silica gel, since the tank design must be adopted.

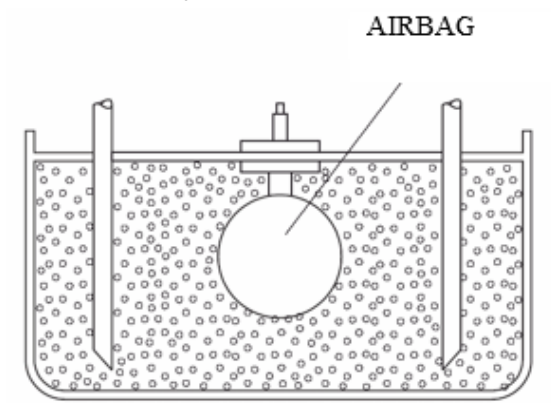

Fig. 4. Basic design of pressurised airbag inserted into the tank

A.L. Hitchcox [8] mentioned that a sealed or pressurized reservoir mentioned earlier could be a solution, in terms of system complexity and costs, air breathers can be more simple solution. Solution that researchers [8] came up is an inflatable bladder. It is made of synthetic fiber coated and vulcanized inside and out with an oil-resistant elastomer. In Fig.5 we can see inflatable bladder mechanism. By moving piston forward, a vacuum is created and bladder inflates with surrounding air, which is completely sealed from being in contact with hydraulic fluid. Therefore contamination with outside moisture and particles is prevented. When piston is moved backwards, the fluid returns to the reservoir, which pushes the air out of the bladder, causing it to deflate. The compensator (bladder) can also be placed outside of reservoir in case valves and other components are in the way. If bladder is placed outside, it can be connected or configured with tubing. One of the pros of system containing compensator is, that there is no need for filter elements, which can clog or get depleted. Compensator or reservoir isolator is essentially maintenance free. This solution is used mainly in cases where the hydraulic tank rotates together with the object, e.g. plane. Thus, the suction pipe of the pump is always below the surface of the liquid. Also with proper bladder material selection, reservoir isolators are compatible with almost any industry field. Only few of them are power generator plants, automotive plants, steel and primary metal fields, any many more highly contaminated or humid environment [2].

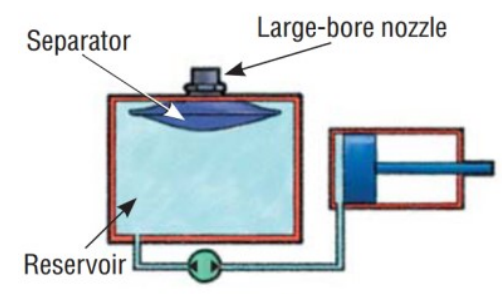

System starts with a full reservoir

Interior of separator is open to atmosphere

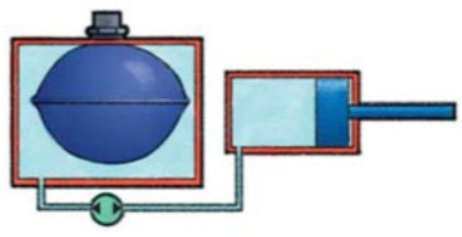

With system running, oil volume in the reservoir is replaced by air volume in the separator

Fig. 5. Volume compensator, which prevent air coming in direct contact with hydraulic fluid

D.D. Troyer [1] also suggest proper selection and good maintenance of the hydraulic wiper seals, which expose risk of contaminant entering the system. 
Reservoir design can also impact oils water shedding characteristics. Water has higher density than hydraulic oil, therefore in case of saturation, water can be in separated phase from oil. It can either be in form of emulsified water or free form water. Since density of water is higher, it tends to float below hydraulic oil, example is shown on Fig.1. If suction line is properly placed, the hydraulic system has reduced chance for water intake, therefore preventing high water contamination in hydraulic system. Other hydraulic components are then also less exposed to water contamination.

It is also important to maintain heat exchangers and coolers, to prevent leakage-related contamination.

We can also prevent water contamination by using modern completely closed hydraulic systems, e.g. self-contained electro-hydraulic cylinders or Electro Hydraulic Actuators. Publication [9] confirms Electro Hydraulic Actuators can be an option, but not complete solution. Authors [9] explained, that even closed hydraulic system have problem with water contamination and that typical saturation for hydraulic oil is $300 \mathrm{ppm}$.

Next method is not water excluding method, but rather water removing method. It is solution that researcher [9] came up. It consists of system pumping oil through a filter, which medium absorbs moisture and also filters particles down to about $30 \mu \mathrm{m}$. Process of filtration produces no frothing. Although it is not water excluding method, it is still option to remove excess water from hydraulic system, if proper water excluding methods from various factors can not be applied.

Research [10] suggest, that exchange of air with environment can also occur in waterproof containers, when those are exposed to wide temperature ranges. Wide temperature ranges cause containers to expand and contract air and liquid inside. New or used containers should therefore be appropriately stored and handled. It is recommended, that container lids are continuously checked for appropriate sealing tightness.

\section{WATER PREVENTION WITH HYDRAULIC SYSTEM CARE}

It is most important to ensure no water/moisture in the hydraulic fluid, but if it is still present, its content should be as low as possible. Even the smallest amount of water affects the chemical properties of the oil and affects the metal surfaces of the hydraulic components and the tank in the device. The level of damage to the oil and the service life of the machine (see Fig.6) depends on the amount and presence of water in the oil. Therefore, the aim must not be to ensure that the water content is below the permissible limit, but to operate the device without or at least with the minimum possible water content.

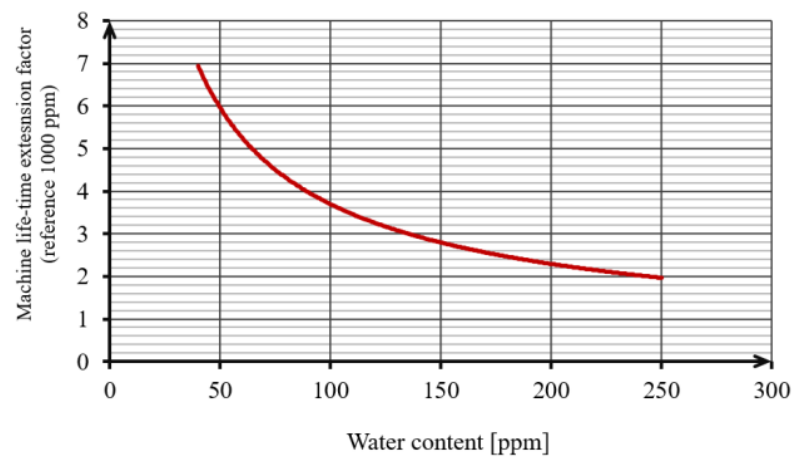

Fig. 6. Predicted hydraulic system extension factor when using hydraulic oil with less then 1000 ppm (reference) water content

Among all previously explained water prevention methods, is also important to take care of hydraulic system. This can be done by regularly checking fluid levels. If fluid level is dropping it can indicate leakage and also poorly sealed system, which can allow enter of moisture. When topping up system, it is recommended to use same hydraulic fluid in terms of brand and viscosity grade. If minimum water contamination is required, new hydraulic fluid should also be stripped off moisture. Water that is dissolved in oil, before it reaches saturation point, can be difficult to remove. In case of external factors, some part of dissolved water in hydraulic fluid, can change form in emulsified droplets, which are causing oxidation and rust. If breathers are installed, breather caps, breather filters and fill screens should be also regularly inspected. Hydraulic system should be visually inspected for potential leakages from hoses, pipes, pipe connections, etc. Checking temperature of system is also important, if temperature is to high, it can indicate cooler defect or leakage. Cooler leakage can also contaminate hydraulic fluid.

\section{CONCLUSION}

Paper explains how important it is to keep water contamination in hydraulic system to minimum. There are different approaches in terms of design and proper hydraulic component selection, to solve water contamination problem. Complete water removal from hydraulic system is 
practically impossible, but it is possible to keep target water level at low relative humidity, which prevents emulsified or free form water, which is very aggressive toward components wear in terms of oxidation and corrosion. Most effective methods of excluding water contamination mentioned are desiccant air breathers or so called Silica Gel filters, for hydraulic system where air from surrounding comes into contact with fluid in reservoir. Or expanding compensators, which are used for closed system and can be inserted into reservoir, or be used as outside units connected via tubing. Expanding compensators and air breathers can also come in different forms and are meant for different environments or application requirements. Good prevention of water contamination should also be appropriate system care, maintenance and regular observation in terms of possible leakages. In terms of water contamination monitoring, there are also few mentioned methods. Seals on the hydraulic system components must also be appropriately chosen and designed for best compatibility, durability and tightness to prevent outside moisture from entering the system.

Based on conducted analysis, we can give following recommendations. For hydraulic tanks operating in an environment with high humidity, the simplest solution is to install a silica gel filter combined with a non-return valve. The size of the silica gel filter should be chosen according to the recommendations of the manufacturer of this filter, taking into account the size of the tank (and thus the space of air above the liquid), the usual level of humidity in the surrounding air and of course the material of the filter.

It is also important to remove solid contaminants, which is most easily achieved by good filtration with continuous on-line monitoring of fluid cleanliness and moisture content. On devices that are crucial for production, operate in $24 / 7$ mode and/or are away from professional maintenance personnel, such a system, according to our many practical experiences, should not be missing. Please note: the downtime of a larger machine represents a much higher cost than the common price of such online monitoring system.

\section{NOTE}

The abstract of this paper is published at the $10^{\text {th }}$ International Conference on Tribology BALKANTRIB '20 organised in Belgrade, on May 2022, 2021.

\section{REFERENCES}

[1] D.D. Troyer, Advanced strategies for the monitoring and control of water contamination in oil hydraulic fluids; research paper in G. Totten, D. Wills, D. Feldmann (Ed.): Symposium on Hydraulic Failure Analysis Fluids, Components, and System Effects, American Society of Testing and Materials, Reno, NV, Vol.1339, pp.214-224, 2001.

[2] A.L. Hitchcox, The ultimate in reservoir breathers?. Hydraulics \& Pneumatics, 60 (4), 2007: 17-18.

[3] R.E. Cantley, The Effect of Water in Lubricating Oil on Bearing Fatigue Life. ASLE transactions, 20 (3), 1977: 244-248.

https://doi.org/10.1080/05698197708982838

[4] D. Lovrec, M. Kambič, Hidravlične tekočine in njihova nega. Fakulteta za strojništvo Maribor, 2007.

[5] R. Zhang, X. Yu, Y. Hu, H. Zang, W. Shu, Active control of hydraulic oil contamination to extend the service life of aviation hydraulic system. The International Journal of Advanced Manufacturing Technology, 96, 2018: 16931704.

https://doi.org/10.1007/s00170-017-0833-9

[6] A. Helwig, G. Müller, S. Paul, Health Monitoring of Aviation Hydraulic Fluids Using Opto-Chemical Sensor Technologies. Chemosensors, 8 (4), 2020: 131. https://doi.org/10.3390/chemosensors 80401 $\underline{31}$

[7] S.D. Anderson, getting control of Contamination. Hydraulics \& Pneumatics, 58 (8), 2005: 54-56.

[8] A.L. Hitchcox, Keep air, dirt, and moisture out of reservoirs. Hydraulics \& Pneumatics, 63 (12), 2010: 12.

[9] L. Selsdon, Autoclude: Taking water from troubled oil. Professional Engineering, 18 (7), 2005: 54.

[10] B. Battat, W. Babcock, Understanding and Reducing the Effects of Contamination on Hydraulic Fluids and Systems. The Amptiac Quarerly, 7 (1), 2003: 11-15. 